\title{
The algebra of diffeomorphisms from the world sheet
}

\author{
Waldemar Schulgin ${ }^{a}$ and Jan Troost ${ }^{b, 1}$ \\ ${ }^{a}$ Université Libre de Bruxelles and International Solvay Institutes, \\ ULB-Campus Plaine CP231, Bruxelles, Belgium \\ ${ }^{b}$ Laboratoire de Physique Théorique, \\ 24 rue Lhomond, 75005 Paris, France \\ E-mail: waldemar.schulgin@ulb.ac.be, jan.troost@lpt.ens.fr
}

\begin{abstract}
The quantum theory of a massless spin two particle is strongly constrained by diffeomorphism invariance, which is in turn implied by unitarity. We explicitly exhibit the space-time diffeomorphism algebra of string theory, realizing it in terms of world sheet vertex operators. Viewing diffeomorphisms as field redefinitions in the two-dimensional conformal field theory renders the calculation of their algebra straightforward. Next, we generalize the analysis to combinations of space-time anti-symmetric tensor gauge transformations and diffeomorphisms. We also point out a left-right split of the algebra combined with a twist that reproduces the C-bracket of double field theory. We further compare our derivation to an analysis in terms of marginal deformations as well as vertex operator algebras.
\end{abstract}

KeYwords: Bosonic Strings, Conformal Field Models in String Theory, Space-Time Symmetries, BRST Symmetry

ARXIV EPRINT: 1407.1385

\footnotetext{
${ }^{1}$ Unité Mixte du CNRS et de l'Ecole Normale Supérieure associée à l'université Pierre et Marie Curie 6, UMR 8549.
} 


\section{Contents}

1 Introduction $\quad 1$

2 The algebra of gauge transformations 3

2.1 Diffeomorphisms in the path integral 3

2.2 Diffeomorphisms as field redefinitions 4

2.3 The algebra of diffeomorphisms 5

2.4 The algebra including anti-symmetric gauge transformations 6

2.5 Contributions 8

3 Comparison to other methods $\quad 8$

3.1 The chiral cohomological Gerstenhaber algebra 9

$\begin{array}{lr}3.2 & \text { The non-chiral Gerstenhaber algebra } \\ 3.3 & 9\end{array}$

$\begin{array}{lll}3.3 & \text { Marginal perturbations } & 10\end{array}$

4 Conclusions $\quad 12$

A Exactness, on-shellness and marginality 13

$\begin{array}{lll}\text { A.1 Diffeomorphisms and anti-symmetric gauge transformations } & 13\end{array}$

$\begin{array}{lll}\text { A.2 An example three-point function } & 13\end{array}$

$\begin{array}{ll}\text { B Factorization and doubling } & 15\end{array}$

\section{Introduction}

String theory is a theory containing a massless spin two particle. There is a classic argument that a consistent interacting theory of a massless spin two particle is necessarily a theory of general relativity in the sense that the non-linear interactions are constrained by the requirement of general coordinate invariance. The gauge symmetry must survive in the quantum theory to guarantee unitarity through the decoupling of unphysical degrees of freedom. ${ }^{1}$ This argument applies to perturbative string theory since it contains a massless spin two particle and is unitary. In the literature, string perturbation theory is more explicitly demonstrated to be diffeomorphism invariant through a combination of two arguments. It is shown that the transformation of the metric under a diffeomorphism corresponds to adding a BRST exact state to the string state at hand. Secondly, BRST exact states decouple from string amplitudes through a generalized canceled propagator argument (based on analyticity of the S-matrix). And therefore, string perturbation theory is diffeomorphism invariant.

\footnotetext{
${ }^{1}$ The history of this argument is reviewed in detail in the foreword to [1] and a version of the argument is contained in the lectures [1].
} 
We desire to go one step further in rendering this classic argument more explicit in the context of string theory. We not only wish to identify the relevant gauge transformations corresponding to infinitesimal coordinate transformations in space-time, but also compute the algebra of diffeomorphisms in terms of world sheet vertex operators. Indeed, the gauge algebra of string theory is much larger than the diffeomorphism algebra (see e.g. [2]). It is interesting per se to isolate in this huge symmetry algebra the gauge algebra governing gravitational interactions and the equivalence principle (see also e.g. [3]).

We have given a general motivation for attacking this problem. We are also inspired by the desire to realize asymptotic symmetry algebras in string theory. See for instance $[4,5]$ and [6-9] for older AdS and more recent flat space applications of an understanding of these asymptotic symmetries on the world sheet. The asymptotic symmetry algebra consist of asymptotically non-trivial diffeomorphisms. A natural prerequisite to understanding their world sheet counterparts is to understand the algebra of diffeomorphisms on the world sheet in detail. A third motivation for our work arises in the typically stringy extension of the algebra of diffeomorphisms to also include anti-symmetric tensor gauge transformations. The combination of the two naturally suggests a split of these space-time gauge transformations in terms of world sheet left- and right-movers. These may play a role in our understanding of double field theory and T-duality (see e.g. [10, 11] for reviews), with potentially interesting applications (e.g. to non-geometric regions of string vacua, or to desingularizing cosmological evolutions).

The paper is organized as follows. In section 2, we show how to interpret space-time diffeomorphisms as field redefinitions in the two-dimensional conformal field theory. We demonstrate in the covariant BRST formalism that the representation of these diffeomorphisms on the world sheet indeed satisfies the expected diffeomorphism algebra. We then add anti-symmetric gauge transformations to the algebra. Their crucial property will be that exact shifts of the anti-symmetric tensor do not influence the world sheet equations of motion, nor the world sheet energy-momentum tensor. That will allow us to explicitly compute the mixed algebra of diffeomorphisms and anti-symmetric gauge transformations from the world sheet as well.

In section 3, we compare our derivation to other approaches. In particular, we connect to the approach in which deformations of the action are treated in conformal perturbation theory. These approaches are related to the calculation of world sheet vertex operator algebras, which mimic the diffeomorphism algebra at the classical level, but also contain $\alpha^{\prime}$ corrections [7, 12, 13].

In the first appendix, we stress that, though operators $c \tilde{c} O^{\text {matter }}$ that we insert in string correlation functions typically need to be of (matter) dimension $(1,1)$, this does not need to hold for pure gauge operators, e.g. for diffeomorphism vertex operators. Those are automatically on-shell.

In the second appendix B, we point out how to factor and twist the algebra of diffeomorphisms and anti-symmetric gauge transformations into an algebra dependent on world sheet left-movers and right-movers independently, and satisfying the C-bracket commutator. 


\section{The algebra of gauge transformations}

In this section, we derive the algebra of diffeomorphisms, and anti-symmetric gauge transformations from a world sheet perspective.

\subsection{Diffeomorphisms in the path integral}

To implement diffeomorphisms in terms of the two-dimensional world sheet theory on the fundamental string propagating in flat space, we can turn to a path integral formalism for the gauge fixed world sheet theory, which gives rise to a two-dimensional conformal field theory. Ignoring world sheet ghosts, we find the path integral:

$$
Z=\int[D X(z, \bar{z})] \exp (-S[X(z, \bar{z})])
$$

where the world sheet action is

$$
S=\frac{1}{2 \pi \alpha^{\prime}} \int d^{2} z \eta_{\mu \nu} \partial X^{\mu} \bar{\partial} X^{\nu},
$$

and where we can allow for operator insertions representing string scattering. We wish to consider the effect of target space diffeomorphisms on the path integral. Diffeomorphisms in the target space can be interpreted on the world sheet as field redefinitions:

$$
X^{\mu}(z, \bar{z}) \rightarrow X^{\mu}(z, \bar{z})+\xi^{\mu}\left(X^{\nu}(z, \bar{z})\right) .
$$

When we perform the field redefinition on the action, we find that to first order, the metric is shifted by the diffeomorphism according to the standard rule. The measure $[D X]$ will transform unless we introduce the traditional measure factor $\sqrt{-G[X]}$ in equation (2.1), where $G[X]$ is the determinant of the space-time metric $G_{\mu \nu}$. Indeed, the measure factor will pick up a Jacobian transformation factor, while the square root of the determinant will transform inversely under diffeomorphisms. When we compute the commutator of gauge transformations, after a first application of a diffeomorphism, the metric becomes nontrivially dependend on the coordinates $X$, and this measure factor will become non-trivial. In a second application of a diffeomorphism then, this measure factor needs to be taken into account.

If we concentrate on the world sheet kinetic term, and compute the algebra of diffeomorphisms, we find the standard algebra - see later for the details:

$$
\left[\xi^{1}, \xi^{2}\right]=\mathcal{L}_{\xi^{1}} \xi^{2}-\mathcal{L}_{\xi^{2}} \xi^{1}
$$

represented on the space of metric couplings in the two-dimensional field theory. To conclude that we realize the standard diffeomorphisms, we must argue that the measure factor $\sqrt{G}$ will not lead to non-trivial contributions to string scattering. In [14], where the above argument can also be found, it is argued that this is the case because of the tracelessness of the graviton polarization tensor. The same type of reasoning that we make above would hold in curved spaces. This is the underlying rationale for claiming that string theory in 
curved space will be diffeomorphism invariant. Anti-symmetric gauge transformations can also be treated in this formal path integral argument.

There are several reasons to proceed beyond this. The first is that one may wish to carefully regularize the path integral. A second reason is that one wants to understand better the space of deformations of the world sheet theory, and in particular distinguish those that are trivial from the target space perspective from those that are not. E.g. one would like to identify BRST exact deformations from others in string theory, through a more direct analysis of the shifts of the string state. Another reason is that one can have diffeomorphisms that do not fall fast enough at infinity to make them trivial field redefinitions. They can then generate a large and interesting asymptotic symmetry group. One may then wish for an explicit realization of the symmetry algebra on the world sheet in terms of charge operators. Any of these motivations lead us to attempt to analyze the above transformations in some more detail from a world sheet perspective.

\subsection{Diffeomorphisms as field redefinitions}

We wish to be more explicit about how to compute the algebra of diffeomorphisms from a world sheet perspective. We start from a world sheet theory on a flat target space, with fields $X^{\mu}$ and metric $\eta_{\mu \nu}$ and action (2.2). The fields $X^{\mu}(z, \bar{z})$ are shifted as in the field redefinition (2.3). The world sheet action after applying the diffeomorphism becomes:

$$
\begin{aligned}
S & =\frac{1}{2 \pi \alpha^{\prime}} \int d^{2} z \eta_{\mu \nu} \partial\left(X^{\mu}+\xi^{\mu}\right) \bar{\partial}\left(X^{\nu}+\xi^{\nu}\right) \\
& \approx \frac{1}{2 \pi \alpha^{\prime}} \int d^{2} z\left(\eta_{\mu \nu}+\partial_{\mu} \xi_{\nu}+\partial_{\nu} \xi_{\mu}\right) \partial X^{\mu} \bar{\partial} X^{\nu}
\end{aligned}
$$

where we always must think of the shift $\xi^{\mu}\left(X^{\nu}\right)$ as field dependent. We work to leading order in the expansion in the deformation $\xi$, and in the above formula, we have used the Minkowksi metric to lower the index on $\xi$. There are now at least two useful perspectives on the shifted action. One that links quickly to the traditional perspective on diffeomorphisms is to view:

$$
\delta_{\xi} S=\frac{1}{2 \pi \alpha^{\prime}} \int d^{2} z\left(\partial_{\mu} \xi_{\nu}+\partial_{\nu} \xi_{\mu}\right) \partial X^{\mu} \bar{\partial} X^{\nu}
$$

as the integrated form of a vertex operator associated to a BRST exact state in the original theory. ${ }^{2}$ When we assume the integrand of the vertex operator to be marginal, this perspective says that we have performed a marginal deformation of the world sheet theory that we could treat in conformal perturbation theory. We will return to this perspective in section 3. A second perspective is to concentrate on the first line of (2.5) and to say that the new world sheet theory is very much like the old one, except for the fact that the field $X^{\mu}$ has been replaced by the field $X^{\mu}+\xi^{\mu}$. In the latter perspective, we draw conclusions like the fact that the operator product expansion in the deformed theory is (at $\alpha^{\prime}=2$ ):

$$
(X+\xi)^{\mu}(z, \bar{z})(X+\xi)^{\nu}(w, \bar{w}) \approx-\eta^{\mu \nu} \log |z-w|^{2},
$$

\footnotetext{
${ }^{2}$ There is a subtlety here in that the integrand of this vertex operator is often assumed to be of world sheet dimension $(1,1)$, although there is no such restriction on $\xi$ from the target space point of view. We discuss this subtlety in appendix A.
} 
and that the metric is still the initial metric $\eta_{\mu \nu}$, while the energy-momentum tensor reads:

$$
T^{m}(\xi)=-\frac{1}{2} \eta_{\mu \nu} \partial(X+\xi)^{\mu} \partial(X+\xi)^{\nu} .
$$

In this perspective, in the BRST covariant treatment, the BRST charge of the new theory is given in terms of the deformed energy-momentum tensor (2.8).

\subsection{The algebra of diffeomorphisms}

Let's now consider these transformations in the covariant BRST formalism. In a first step, we have an undeformed BRST vertex operator:

$$
Q_{B}(0)=\frac{1}{2 \pi i} \oint\left(d z j_{B}-d \bar{z} \tilde{j}_{B}\right)
$$

where the ghost currents $j_{B}$ and $\tilde{j}_{B}$ depend on the undeformed world sheet energymomentum tensor. We perform a shift of the background by a BRST exact operator that is obtained by computing the commutator of the undeformed BRST charge with a first seed ${ }^{3}$ operator $s_{1}$, depending on a parameter $\xi_{1}$ :

$$
s_{1}=\tilde{c} \xi_{\mu}^{1} \bar{\partial} X^{\mu}-c \xi_{\mu}^{1} \partial X^{\mu} .
$$

When we compute the commutator, we find the BRST exact operator:

$$
\begin{aligned}
q= & {\left[Q_{B}(0), s_{1}\left(\xi_{1}\right)\right] } \\
= & \left(\partial_{\mu} \xi_{\nu}^{1}+\partial_{\nu} \xi_{\mu}^{1}\right) c \tilde{c} \partial X^{\mu} \bar{\partial} X^{\nu} \\
& \quad+\text { other (left,right) ghost structures . }
\end{aligned}
$$

The shift of the background by this operator shifts the metric coupling of the background according to the rule:

$$
\eta_{\mu \nu} \rightarrow \eta_{\mu \nu}+\left(\partial_{\mu} \xi_{\nu}^{1}+\partial_{\nu} \xi_{\mu}^{1}\right)
$$

thus implementing the first diffeomorphism.

To compute the commutator of two diffeomorphisms, we wish to perform a second BRST exact shift of the background. The BRST-charge after one deformation is given by

$$
Q_{B}\left(\xi^{1}\right)=\frac{1}{2 \pi i} \oint\left(d z j_{B}-d \bar{z} \tilde{j}_{B}\right)
$$

with matter energy-momentum tensor depending on $\xi^{1}$ as in equation (2.8)

$$
j_{B}=c T^{\mathrm{m}}\left(\xi^{1}\right)+: b c \partial c:+\frac{3}{2} \partial^{2} c .
$$

The second seed that we wish to consider is most naturally written in terms of the deformed fields $X^{\mu}+\xi^{1 \mu}$, such that we find - this should be compared to (2.10):

$$
s_{2}=\tilde{c} \xi^{2}\left(X+\xi^{1}\right) \cdot \bar{\partial}\left(X+\xi^{1}\right)-c \xi^{2}\left(X+\xi^{1}\right) \cdot \partial\left(X+\xi^{1}\right) .
$$

\footnotetext{
${ }^{3}$ The seed is the state on which we act with the BRST operator to obtain a BRST exact, or pure gauge state. It can be thought off as the gauge parameter.
} 
The contraction is done with the flat background metric - remember that we took the perspective in which the metric is not shifted after the first deformation. The calculation of the BRST exact state that results from this seed is identical to the standard calculation in flat space (thanks to exact operator product expansions like (2.7)), with the mere replacement of the field $X$ by $X+\xi^{1}$, and we therefore find the result:

$$
\begin{aligned}
C_{12}= & {\left[Q_{B}\left(\xi^{1}\right), s_{2}\right] } \\
= & c \tilde{c}\left(\partial_{\mu} \xi_{\nu}^{2}\left(X+\xi^{1}\right)+\partial_{\nu} \xi_{\mu}^{2}\left(X+\xi^{1}\right)\right) \partial\left(X+\xi^{1}\right)^{\mu} \bar{\partial}\left(X+\xi^{1}\right)^{\nu} \\
& + \text { other ghost structures }
\end{aligned}
$$

Finally, we are ready to compute the result of the commutator of two diffeomorphisms. We take into account the linear shift in the background in $\xi^{1}$, and anti-symmetrize the above expression in the deformations labeled 1 and 2, which results in the commutator:

$$
\begin{aligned}
C= & {\left.\left[Q_{B}(0), s_{1}\right]+\left[Q_{B}\left(\xi^{1}\right), s_{2}\right)\right]-(1 \leftrightarrow 2) } \\
= & c \tilde{c}\left(\partial_{\mu}\left(\xi^{1 \rho} \partial_{\rho} \xi_{\nu}^{2}-\xi^{2 \rho} \partial_{\rho} \xi^{1 \nu}\right)+\partial_{\nu}\left(\xi^{1 \rho} \partial_{\rho} \xi_{\mu}^{2}-\xi^{2 \rho} \partial_{\rho} \xi_{\mu}^{1}\right)\right) \partial X^{\mu} \bar{\partial} X^{\nu} \\
& + \text { other ghost structures } .
\end{aligned}
$$

This is the BRST exact shift of the background metric that results from consecutive gauge transformations 1 and 2, minus 2 then 1 . Through expansions in the shifts $\xi^{1}$ and $\xi^{2}$, we confirm the expected result (2.4). With field redefinitions as our guide, the calculation of the commutator in the two-dimensional quantum field theory has become a close analogue of its classical, geometric counterpart.

\subsection{The algebra including anti-symmetric gauge transformations}

Anti-symmetric gauge transformations in string theory are closely related to diffeomorphisms. Under T-duality, the two types of gauge symmetries become equivalent. It is therefore natural to attempt to treat them on the same footing. Still, in the canonical treatment they need separate care. Let's analyze how they modify the picture drawn for diffeomorphisms in subsection 2.3. We can introduce anti-symmetric gauge transformations as follows. In the first perturbation, we consider also a shift of the action which is a total derivative on the world sheet:

$$
\begin{aligned}
\delta_{\tilde{\xi}} S & =-\frac{i}{2 \pi \alpha^{\prime}} \int d\left(\tilde{\xi}_{\mu} \bar{\partial} X^{\mu} d \bar{z}+\partial X^{\mu} \tilde{\xi}_{\mu} d z\right) \\
& =\frac{1}{2 \pi \alpha^{\prime}} \int d^{2} z\left(\partial \tilde{\xi}_{\mu} \bar{\partial} X^{\mu}-\partial X^{\mu} \bar{\partial} \tilde{\xi}_{\mu}\right) .
\end{aligned}
$$

where in the first line $d$ denotes the exterior derivative applied to the pull-back of the oneform $\tilde{\xi}$ to the world sheet. Since this perturbation is a total derivative on the world sheet, it won't affect the world sheet propagator, energy-momentum tensor and so on. When we perform the analysis of the second shift, using the covariant BRST formalism, we must also generalize the seed to include anti-symmetric tensor gauge transformations:

$$
s_{2}\left(\xi^{2}, \tilde{\xi}^{2}\right)=\tilde{c}\left(\xi^{2}+\tilde{\xi}^{2}\right) \cdot \bar{\partial}\left(X+\xi^{1}\right)-c\left(\xi^{2}-\tilde{\xi}^{2}\right) \cdot \partial\left(X+\xi^{1}\right) .
$$


The second BRST exact shift will then be equal to:

$$
\begin{aligned}
C_{12}= & {\left[Q_{B}\left(\xi^{1}\right), s_{2}\left(\xi^{2}, \tilde{\xi}^{2}\right)\right] } \\
= & c \tilde{c}\left(\partial_{\mu}\left(\xi_{\nu}^{2}\left(X+\xi^{1}\right)+\tilde{\xi}_{\nu}^{2}\left(X+\xi^{1}\right)\right)\right. \\
& \left.+\partial_{\nu}\left(\xi_{\mu}^{2}\left(X+\xi^{1}\right)-\tilde{\xi}_{\mu}^{2}\left(X+\xi^{1}\right)\right)\right) \partial\left(X^{\mu}+\xi^{1 \mu}\right) \bar{\partial}\left(X^{\nu}+\xi^{1 \nu}\right) \\
& + \text { other ghost structures } .
\end{aligned}
$$

The commutator of two gauge transformations is equal to:

$$
\begin{aligned}
C= & {\left[Q_{B}(0), s_{1}\left(\xi^{1}, \tilde{\xi}^{1}\right)\right]+\left[Q_{B}\left(\xi^{1}\right), s_{2}\left(\xi^{2}, \tilde{\xi}^{2}\right)\right]-(1 \leftrightarrow 2) } \\
= & c \tilde{c}\left(\partial_{\mu}\left(\xi^{1 \rho} \partial_{\rho} \xi_{\nu}^{2}-\xi^{2 \rho} \partial_{\rho} \xi^{1 \nu}\right)+\partial_{\nu}\left(\xi^{1 \rho} \partial_{\rho} \xi_{\mu}^{2}-\xi^{2 \rho} \partial_{\rho} \xi^{1 \mu}\right)\right) \partial X^{\mu} \bar{\partial} X^{\nu} \\
& +c \tilde{c}\left(\partial_{\mu}\left(\xi^{1 \rho} \partial_{\rho} \tilde{\xi}_{\nu}^{2}-\xi^{2 \rho} \partial_{\rho} \tilde{\xi}^{1 \nu}\right)-\partial_{\nu}\left(\xi^{1 \rho} \partial_{\rho} \tilde{\xi}_{\mu}^{2}-\xi^{2 \rho} \partial_{\rho} \tilde{\xi}^{1 \mu}\right)\right) \partial X^{\mu} \bar{\partial} X^{\nu} \\
& +c \tilde{c}\left(-\partial_{\mu} \xi^{1 \rho} \partial_{\nu} \tilde{\xi}_{\rho}^{2}+\partial_{\nu} \xi^{1 \rho} \partial_{\mu} \tilde{\xi}_{\rho}^{2}+\partial_{\mu} \xi^{2 \rho} \partial_{\nu} \tilde{\xi}_{\rho}^{1}-\partial_{\nu} \xi^{2 \rho} \partial_{\mu} \tilde{\xi}_{\rho}^{1}\right) \partial X^{\mu} \bar{\partial} X^{\nu} \\
& + \text { other ghost structures }
\end{aligned}
$$

If we use the notations $D(\xi)$ for target space diffeomorphisms and $A(\xi)$ for antisymmetric tensor gauge transformations when they are represented on the metric and anti-symmetric tensor couplings in the world sheet action, we can summarize the algebra of these generators: ${ }^{4}$

$$
\begin{aligned}
{\left[D\left(\xi^{1}\right), D\left(\xi^{2}\right)\right] } & =D\left(\left[\xi^{1}, \xi^{2}\right]\right) \\
{\left[A\left(\tilde{\xi}^{1}\right), A\left(\tilde{\xi}^{2}\right)\right] } & =0 \\
{\left[D\left(\xi^{1}\right), A\left(\tilde{\xi}^{2}\right)\right] } & =A\left(\mathcal{L}_{\xi^{1}} \tilde{\xi}^{2}\right) .
\end{aligned}
$$

We have the freedom to add a total derivative term to the argument of the anti-symmetric tensor gauge transformation in the last line on the right hand side — see e.g. [10] for a clear discussion - to obtain the Courant bracket (with extra automorphism symmetry):

$$
\begin{aligned}
& {\left[D\left(\xi^{1}\right), D\left(\xi^{2}\right)\right]=D\left(\left[\xi^{1}, \xi^{2}\right]\right)} \\
& {\left[A\left(\tilde{\xi}^{1}\right), A\left(\tilde{\xi}^{2}\right)\right]=0} \\
& {\left[D\left(\xi^{1}\right), A\left(\tilde{\xi}^{2}\right)\right]=A\left(\mathcal{L}_{\xi^{1}} \tilde{\xi}^{2}-\frac{1}{2} d\left(\xi^{1} \cdot \tilde{\xi}^{2}\right)\right) .}
\end{aligned}
$$

In appendix $\mathrm{B}$, we demonstrate the existence of an algebra which is factorized in the leftand right-movers on the world sheet. The algebra has a commutator equal to the C-bracket algebra of $[10,15,16]$.

\footnotetext{
${ }^{4}$ We have $\left(\mathcal{L}_{\xi^{1}} \tilde{\xi}^{2}\right)_{\mu}=\xi^{1 \rho} \partial_{\rho} \tilde{\xi}_{\mu}^{2}+\partial_{\mu} \xi^{1 \rho} \tilde{\xi}_{\rho}^{2}$ since $\tilde{\xi}$ is thought off as a one-form.
} 


\subsection{Contributions}

We want to make a brief remark on the terms that survive in the final result. For simplicity, we concentrate on the diffeomorphism algebra. If we denote by $Y=X+\xi$ the redefined field, and attach an index $Y$ to the quantities in the redefined frame, then we find the following relation between the BRST exact state $q^{Y}$ in the $Y$ basis and the BRST exact states $q^{X}$ in the $X$ basis:

$$
q^{Y}\left(\xi^{2}\right)=q^{X}\left(\xi^{2}\right)+q^{X}\left(\xi^{1 \rho} \partial_{\rho} \xi^{2}\right)+\text { terms symmetric in } \xi^{1} \text { and } \xi^{2} .
$$

The commutator of diffeomorphisms is then given by the usual algebra of vector fields, in the original $X$ frame:

$$
q^{X}\left(\xi^{1}\right)+q^{Y}\left(\xi^{2}\right)-q^{X}\left(\xi^{2}\right)-q^{Y}\left(\xi^{1}\right)=q^{X}\left(\left[\xi^{1}, \xi^{2}\right]\right) .
$$

We see that only the second term in equation (2.24) survives in the end result. Yet a third way to present the calculation is to rewrite the BRST exact term in the $Y$-frame in terms of variations of the BRST charge, the seed, as well as the operator product expansion:

$$
\begin{aligned}
q^{Y}\left(\xi^{2}\right)= & {\left.\left[Q_{B}^{Y}, s^{Y}\left(\xi^{2}\right)\right]\right|_{O P E^{Y}} } \\
= & {\left.\left[Q_{B}^{X}+\delta_{\xi^{1}} Q_{B}^{X}, s^{X}\left(\xi^{2}\right)+\delta_{\xi^{1}} s^{X}\left(\xi^{2}\right)\right]\right|_{O P E^{X}} } \\
+ & {\left.\left[Q_{B}^{X}+\delta_{\xi^{1}} Q_{B}^{X}, s^{X}\left(\xi^{2}\right)+\delta_{\xi^{1}} s^{X}\left(\xi^{2}\right)\right]\right|_{\xi_{\xi^{1}} O P E^{X}} } \\
= & {\left.\left[Q_{B}^{X}, s^{X}\left(\xi^{2}\right)\right]\right|_{O P E^{X}}+\left.\left[Q_{B}^{X}, \delta_{\xi^{1}} s^{X}\left(\xi^{2}\right)\right]\right|_{O P E^{X}} } \\
& +\left.\left[\delta_{\xi^{1}} Q_{B}^{X}, s^{X}\left(\xi^{2}\right)\right]\right|_{O P E^{X}}+\left.\left[Q_{B}^{X}, s^{X}\left(\xi^{2}\right)\right]\right|_{\xi_{\xi^{1}} O P E^{X}}
\end{aligned}
$$

We have for instance used the notation $\delta_{\xi^{1}} O P E^{X}$ to indicate that after deformation, the operator product expansions for the field $X$ have shifted, et cetera. Once we consider the commutator action of the diffeomorphisms all the terms except the contribution from the second term will sum to zero. This is a version of the observation in equation (2.24) in conformal perturbation theory, namely that the crucial term is a variation of the first diffeomorphism parameter with respect to the second, and that other variations in the perturbation series drop out.

\section{Comparison to other methods}

In this section, we wish to compare our explicit world sheet identification of the diffeomorphism algebra inside the large gauge algebra of string theory to various algebraic structures that have been identified in string theory in the past. We indicate where these structures relate to the algebra we have exhibited, and how they differ. We permit ourselves to wander slightly, in order to overlap with various approaches. Our stroll illustrates that the smooth sailing we experienced in section 2 was due to our choice of route.

The literature has mostly concentrated on (physical or pure gauge) deformations of the world sheet theory in terms of conformal perturbation theory. We can fit an action 
deformed by a diffeomorphism in this category by viewing the shift of the action under the field redefinition $X \rightarrow X+\xi$ as a deformation of the original theory, and performing perturbation theory for the correlators:

$$
\langle\ldots\rangle_{\xi}=\sum_{n=0}^{\infty}\left\langle\frac{1}{n !}\left(\frac{1}{2 \pi \alpha^{\prime}} \int d^{2} z(\partial \xi \cdot \bar{\partial} X+\partial X \cdot \bar{\partial} \xi)\right)^{n} \ldots\right\rangle_{0} .
$$

All correlators in the perturbed theory obtain contributions from the extra insertions. The technique we used in section 2 boils down to an elementary, effortless resummation of conformal perturbation theory, available only in the case where this deformation is equivalent to a field redefinition. If one performs several consecutive deformations, one can ask whether the algebra of deforming vertex operators (as in equation (3.1)) is related to the diffeomorphism algebra we found in section 2. We will address this question in this section, and connect it to various approaches in the literature.

\subsection{The chiral cohomological Gerstenhaber algebra}

In [17], BRST algebraic structures in chiral or open bosonic string theory were discussed. In particular, the compatibility between holomorphic normal ordering, the $b_{-1}$ curly bracket operation and the chiral BRST cohomology were analyzed (see [17] for details). It was shown that they form a Gerstenhaber algebra in cohomology. We can read the relevant proofs of these properties slightly differently, namely, in the full covariant Hilbert space. The proofs of [17] then say that if two states are BRST exact, their curly bracket gives again an exact state, and the seed can be explicitly calculated. Similarly, if three states are BRST exact, their curly bracket Jacobiator is exact, and the seed of the Jacobiator can be explicitly calculated. These are interesting properties of the chiral gauge algebra in string theory. They are closely related to properties of (chiral) vertex operator algebras reviewed for instance in [18] and exploited for chiral analogues of generalized diffeomorphism vertex operator algebras in $[7,12,13]$. However, to usefully compare to our results, we need to study a non-chiral version of these algebras.

\subsection{The non-chiral Gerstenhaber algebra}

The curly bracket operation and its relation to the BRST operator, and marginal deformations has been further extended and exploited in the non-chiral context, e.g. in [19]. The deformation of a matter conformal field theory by a marginal operator was written in [19] in the language of the algebraic structures of [17], generalized to the non-chiral setting. In particular, this requires the introduction of a non-chiral form of normal ordering which was chosen to be:

$$
N O\left(V_{1}, V_{2}\right)(0)=P_{0}\left(V_{1}(\epsilon) V_{2}(0)\right)
$$

where $P_{0}$ is a projection operator on the term in the operator product expansion between $V_{1}$ and $V_{2}$ that is independent of $\epsilon$, including its phase. Effectively, the normal ordering is taken to project onto the non-singular term in both the holomorphic and the antiholomorphic factor separately. The non-chiral curly bracket operation acts as a chiral 
curly bracket on one side, times a normal ordering operation on the other. One then again identifies the resulting algebra as a Gerstenhaber algebra. (See [17] and [19] for details.) For a recent analysis of how one recuperates diffeomorphisms and anti-symmetric gauge transformations within this framework, see [20].

\subsection{Marginal perturbations}

An alternative way to link some of these interesting algebraic structures to our elementary treatment is the following. We need to suppose henceforth that the diffeomorphism operator by which we deform the action is marginal. ${ }^{5}$ A perturbation of the action with the integral of a marginal $(1,1)$ vertex operator $W^{(1,1)}$ corresponds to a deformation of the BRST charge of the form:

$$
Q_{B}^{\text {def }}(z)(\cdot)=Q_{B} \cdot+\{W, \cdot\}
$$

where $W^{(1,1)}$ the doubly descended form of the operator $W=c \tilde{c} W^{(1,1)}$. We have introduced the curly bracket operation $\{.,$.$\} which underlies the Gerstenhaber algebra. { }^{6}$ If we apply this knowledge to a diffeomorphism/anti-symmetric gauge transformation of the form:

$$
W^{(1,1)}=c \tilde{c}\left(\partial \xi^{L} \cdot \bar{\partial} X+\bar{\partial} \xi^{R} \cdot \partial X\right)
$$

then we find the first order deformation:

$$
Q_{B}^{d e f}=Q_{B}+\left\{c \tilde{c}\left(\partial \xi^{L} \cdot \bar{\partial} X+\bar{\partial} \xi^{R} \cdot \partial X\right), \cdot\right\}
$$

The curly bracket replaces the first entry by the operator corresponding to acting by descent once on the operator $c \tilde{c} W^{(1,1)}$, and takes a small contour integral of this operator around the one it is acting on, with the $\epsilon$ regularization prescription described of equation (3.2) (see [19]). We therefore have:

$$
Q_{B}^{d e f}=Q_{B}+\frac{1}{2 \pi i} \oint_{C_{\epsilon}}(d z c-d \bar{z} \tilde{c})\left(\partial \xi^{L} \cdot \bar{\partial} X+\bar{\partial} \xi^{R} \cdot \partial X\right) .
$$

From the result (3.5), we conclude that the description of marginal deformations in the formalism of $[17,19]$ is equivalent to the analysis of the influence of marginal deformations on the BRST charge performed in [21].

The analysis of [21] is based on a regularization procedure in conformal field theory that cuts out little disks from the integral over the world sheet of the marginal deformation of the theory whenever one encounters another vertex operator insertion. The coordinate independence of the deformation of the BRST charge has been analyzed in [22]. That analysis also showed the equivalence between the deformation of [21] and the equal-time contour prescription of the deformation of the world sheet energy-momentum tensor proposed in [14, 23]. Further developments can amongst others be found in [24-26].

\footnotetext{
${ }^{5}$ We remind the reader that this is not generic, as reviewed in appendix A.

${ }^{6}$ Since we hardly need any of its properties, we will not fully review this operation $[17,19]$.
} 
The deformation of the BRST charge is obtained by analyzing the conformal Ward identities in the perturbed theory [21], or by analyzing how conformal transformations influence the procedure of cutting out disks around other vertex operator insertions [22]. Our method in section 2 directly resums conformal perturbation theory. Of course, the methods of $[21,22]$ were designed to be more general, and to apply to physical deformations.

Now that we have reviewed how the deformation of the BRST charge under marginal deformations is coded in a deformation of the world sheet stress-energy tensor of the equaltime contour form discussed in [14,23] we can apply the result of [14] where the deformation of the energy-momentum tensor is interpreted as a change in the background fields, e.g. the metric. ${ }^{7}$ For a given deformation of the background metric $h_{\mu \nu}$, we have the relation [14]:

$$
T\left(h_{\mu \nu}\right)+i\left[\Lambda(\xi), T\left(h_{\mu \nu}\right)\right]=T\left(h_{\mu \nu}+\delta_{\xi} h_{\mu \nu}\right)
$$

between the shifted energy-momenum tensor, the vertex operator $\Lambda(\xi)$ corresponding to the diffeomorphism, and the resulting perturbed energy-momentum tensor. The commutator in equation (3.6) is an equal time commutator. The generator of diffeomorphisms $\Lambda(\xi)$ was proposed to be the vertex operator:

$$
\Lambda(\xi)=\frac{1}{2 \pi i \alpha^{\prime}} \int d \bar{z} \xi_{\mu}(z, \bar{z}) \bar{\partial} X^{\mu}-\frac{1}{2 \pi i \alpha^{\prime}} \int d z \xi_{\mu}(z, \bar{z}) \partial X^{\mu}
$$

with integrands of dimension $(1,0)$ and $(0,1)$, which imposes the conditions:

$$
\partial_{\mu} \xi^{\mu}=0, \quad \partial_{\mu} \partial^{\mu} \xi^{\nu}=0
$$

Now consider an undeformed background with $T(0)=-\frac{1}{2} \eta_{\mu \nu} \partial X^{\mu} \partial X^{\nu}$ and shift the stressenergy tensor by transformations generated by $\Lambda\left(\xi^{1}\right)$ and $\Lambda\left(\xi^{2}\right)$. With a slight abuse of notation, we find:

$$
\begin{aligned}
\delta_{\xi^{2}} \delta_{\xi^{1}} T(0) & =T\left(\xi^{1}\right)+i\left[\Lambda\left(\xi^{2}\right), T\left(\xi^{1}\right)\right] \\
& =T(0)+i\left[\Lambda\left(\xi^{1}\right), T(0)\right]+i\left[\Lambda\left(\xi^{2}\right), T(0)\right]-\left[\Lambda\left(\xi^{2}\right),\left[\Lambda\left(\xi^{1}\right), T(0)\right]\right] .
\end{aligned}
$$

The commutator is then equal to:

$$
\left(\delta_{\xi^{2}} \delta_{\xi^{1}}-\delta_{\xi^{1}} \delta_{\xi^{2}}\right) T(0)=\left[T(0),\left[\Lambda\left(\xi^{2}\right), \Lambda\left(\xi^{1}\right)\right]\right] .
$$

To obtain this result we used the Jacobi identity for the energy-momentum tensor and the operators $\Lambda\left(\xi^{i}\right)$. In [7] we computed the vertex operator algebra between $\Lambda\left(\xi^{1}\right)$ and $\Lambda\left(\xi^{2}\right)$

$$
\left[\Lambda\left(\xi^{1}\right), \Lambda\left(\xi^{2}\right)\right]=\Lambda\left(\xi^{1 \rho} \partial_{\rho} \xi_{\mu}^{2}-\xi^{2 \rho} \partial_{\rho} \xi_{\mu}^{1}-\frac{1}{\alpha^{\prime}}\left(\partial_{\rho} \xi^{1 \nu} \partial_{\mu} \partial_{\nu} \xi^{2 \rho}-\partial_{\rho} \xi^{2 \nu} \partial_{\mu} \partial_{\nu} \xi^{1 \rho}\right)\right)
$$

The equal time commutator between the $\Lambda\left(\xi^{i}\right)$ generates the target space diffeomorphism algebra at leading order in $\alpha^{\prime}$. The vertex operator algebra of the $\Lambda\left(\xi^{i}\right)$ operators does have

\footnotetext{
${ }^{7}$ See also [21] for a analysis of how deformations of the BRST charges due to marginal deformations and due to shifts in the string field are related.
} 
$\alpha^{\prime}$ corrections [7], making the map between the diffeomorphism algebra and the vertex operator algebra an isomorphism only classically. ${ }^{8}$ From this perspective a proper map of the diffeomorphism action from the target space into the world sheet will include $\alpha^{\prime}$-corrections, namely, it is more subtle than the map we used in equation (3.7). It is important to note that the analysis of the vertex operator algebra does not take into account effects such as the deformation of one seed by the other, nor does it take into account possible corrections to the operator product relations after a single deformation (see subsection 2.5). Rather, the vertex operator algebra analysis should be viewed as taking place within conformal perturbation theory.

\section{Conclusions}

String theory is a unitary perturbative theory of quantum gravity. As such we expect it to be diffeomorphism invariant on the basis of classic arguments [1]. We have explicitly exhibited the diffeomorphism algebra of string theory through the interpretation of target space diffeomorphisms as world sheet field redefinitions. This allowed us to efficiently derive the space-time algebra from the world sheet quantum field theory defining perturbative string theory. The derivation of the full diffeomorphism algebra in a fully regularized setting is new, and allows to demonstrate explicitly that there are no $\alpha^{\prime}$ corrections in the gauge algebra we identified. Thus we have exhibited the canonical, undeformed diffeomorphism algebra in the quantum world sheet string theory. We also compared our derivation to algebraic structures in the literature, and showed the subtlety of the comparison to vertex operator algebras which do exhibit $\alpha^{\prime}$ corrections.

We believe these steps are useful in the enterprise of understanding better asymptotic symmetry algebras in string theory, consequences of diffeomorphisms for graviton scattering amplitudes, constraints on doubled effective field theories, et cetera. Our set-up, suitably regularized, is also a good template for similar analyses in curved space, including asymptotic symmetry algebras in asymptotically anti-de Sitter, linear dilaton, de Sitter, or less symmetric space-times.

\section{Acknowledgments}

We would like to thank Costas Bachas, Glenn Barnich, Ben Craps, Giusseppe Policastro, Ashoke Sen and Anton Zeitlin for useful discussions and correspondence. The work of WS was partially supported by the ERC Advanced Grant "SyDuGraM", by a Marina Solvay fellowhip, by IISN-Belgium (convention 4.4514.08) and by the "Communauté Française de Belgique" through the ARC program.

\footnotetext{
${ }^{8} \mathrm{An}$ alternative set of $\alpha^{\prime}$ corrections to the diffeomorphism and anti-symmetric gauge transformation algebra was identified in $[13,27]$ on the basis of a chiral vertex operator algebra [12] in the doubled field theory formalism. These $\alpha^{\prime}$ corrections are consistent with the Green-Schwarz mechanism in heterotic string theory.
} 


\section{A Exactness, on-shellness and marginality}

Often in the string theory literature, on-shellness of a vertex operator and its marginality are viewed as synonyms. Indeed, a matter vertex operator integrand should be marginal in order for the integrated vertex operator to be conformally invariant. A related fact is that a primary operator of the form $c \tilde{c} O_{\text {matter }}$ is BRST closed only if the matter operator $O_{\text {matter }}$ is of dimensions $(1,1)$.

However, there is a slightly more general concept of on-shellness which is important in the context of pure gauge degrees of freedom. Indeed, we can define an operator to be on-shell if is it BRST closed. It is clear then that any world sheet vertex operator that is BRST exact is BRST closed, and therefore on-shell. No condition on its dimension or primary nature are necessary. In other words, pure gauge degrees of freedom are always on-shell. This is logical in the sense that we don't expect pure gauge degrees of freedom to be subject to a physical equation of motion (imposed by the requirement of having a particular conformal dimension). (See [2] for how these facts play out in the larger context of string field theory.)

Since this feature of pure gauge degrees of freedom is often set aside, we wish to treat it in some detail in this appendix. We illustrate this feature with an elementary example, in particular as it pertains to the diffeomorphisms and anti-symmetric gauge transformations discussed in the bulk of the paper.

\section{A.1 Diffeomorphisms and anti-symmetric gauge transformations}

Let's consider a flat target space with the standard BRST operator, energy-momentum tensor, ghost system, et cetera [28]. We consider a seed of the form: ${ }^{9}$

$$
s=\tilde{c} \xi_{\mu}^{L} \bar{\partial} X^{\mu}-c \xi_{\mu}^{R} \partial X^{\mu} .
$$

Acting with the BRST operator gives rise to the BRST exact state:

$$
\begin{aligned}
q= & \left(\partial_{\mu} \xi_{\nu}^{L}+\partial_{\nu} \xi_{\mu}^{R}\right) c \tilde{c} \partial X^{\mu} \bar{\partial} X^{\nu} \\
& -\frac{\alpha^{\prime}}{4}\left(\partial_{\mu} \partial^{\mu} \xi_{\nu}^{L} \partial c \tilde{c} \bar{\partial} X^{\nu}+\partial_{\mu} \partial^{\mu} \xi_{\nu}^{R} c \bar{\partial} \tilde{c} \partial X^{\nu}\right) \\
& + \text { other (left,right) ghost numbers }
\end{aligned}
$$

where we dropped terms that are not of ghost numbers $(1,1)$. Of course, one can act on the full vertex operator (A.2) once more to demonstrate it is BRST closed without having to impose any conditions on the parameters $\left(\xi^{L}, \xi^{R}\right)$.

\section{A.2 An example three-point function}

Let's turn to an example in which we can explicitly see how a non-marginal matter part of a vertex operator leads to a well-defined result for the pure gauge correlator, namely zero. We show that a BRST exact massless vertex operator decouples from a three-point function in

\footnotetext{
${ }^{9}$ See also [3] for an analysis of more generic seed states.
} 
string theory, through explicit computation. A standard technique to demonstrate this fact is to use the contour argument, that has the BRST charge act on the other two insertions of the three-point function (see e.g. [28]). We find the explicit calculation to be informative as well. We calculate the three-point function of a Fourier mode of the BRST exact operator $q$ of equation (A.2)

$$
\begin{aligned}
q\left(k_{1}\right)= & \left(i k_{\mu}^{1} e_{\nu}^{L}\left(k_{1}\right)+i k_{\nu}^{1} e_{\mu}^{R}\left(k_{1}\right)\right) c \tilde{c} \partial X^{\mu} \bar{\partial} X^{\nu} \\
& +\frac{\alpha^{\prime}}{4} k_{1}^{2}\left(e_{\mu}^{L} \partial c \tilde{c} \bar{\partial} X^{\mu}+e_{\mu}^{R} c \bar{\partial} \tilde{c} \partial X^{\mu}\right) \\
& + \text { other (left,right) ghost numbers }
\end{aligned}
$$

with two physical tachyon vertex operator insertions - we follow the conventions of [28] closely:

$$
\begin{aligned}
& S_{3}=\langle:\left.q\left(k_{1}\right)\left(z_{1}, \bar{z}_{1}\right):: c \tilde{c} e^{i k_{2} \cdot X\left(z_{2}, \bar{z}_{2}\right)}:: c \tilde{c} e^{i k_{3} \cdot X\left(z_{3}, \bar{z}_{3}\right)}:\right\rangle \\
&=\left|z_{12} z_{13} z_{23}\right|^{2}\left|z_{12}\right|^{\alpha^{\prime} k_{1} \cdot k_{2}}\left|z_{13}\right|^{\alpha^{\prime} k_{1} \cdot k_{3}}\left|z_{23}\right|^{\alpha^{\prime} k_{2} \cdot k_{3}} \\
& \quad \times\left[\left(i k_{\mu}^{1} e_{\nu}^{L}+i k_{\nu}^{1} e_{\mu}^{R}\right)\left(-i \frac{\alpha^{\prime}}{2}\right)^{2}\left(\frac{k_{2}^{\mu}}{z_{1}-z_{2}}+\frac{k_{3}^{\mu}}{z_{1}-z_{3}}\right)\left(\frac{k_{2}^{\nu}}{\bar{z}_{1}-\bar{z}_{2}}+\frac{k_{3}^{\nu}}{\bar{z}_{1}-\bar{z}_{3}}\right)\right. \\
& \quad+\left(-i \frac{\alpha^{\prime}}{2}\right) \frac{\alpha^{\prime} k_{1}^{2}}{4} e_{\mu}^{L}\left(\frac{1}{z_{1}-z_{2}}+\frac{1}{z_{1}-z_{3}}\right)\left(\frac{k_{2}^{\mu}}{\bar{z}_{1}-\bar{z}_{2}}+\frac{k_{3}^{\mu}}{\bar{z}_{1}-\bar{z}_{3}}\right) \\
&\left.\quad+\left(-i \frac{\alpha^{\prime}}{2}\right) \frac{\alpha^{\prime} k_{1}^{2}}{4}\left(\frac{k_{2}^{\mu}}{z_{1}-z_{2}}+\frac{k_{3}^{\mu}}{z_{1}-z_{3}}\right) e_{\mu}^{R}\left(\frac{1}{\bar{z}_{1}-\bar{z}_{2}}+\frac{1}{\bar{z}_{1}-\bar{z}_{3}}\right)\right] .
\end{aligned}
$$

We left out the overall normalization factor (equal to $2 i g_{c l}^{3} C_{S^{2}} / \alpha^{\prime}$ ) as well as the overall delta-function corresponding to momentum conservation $(2 \pi)^{26} \delta^{26}\left(\sum_{i=1}^{3} k_{i}^{\mu}\right)$. We now use momentum conservation to determine the overall factor for both the $e_{L}$ and $e_{R}$ dependence:

$$
\alpha^{\prime}\left(2 k_{1} \cdot k_{2}+k_{1}^{2}\right)=\alpha^{\prime}\left(k_{3}^{2}-k_{2}^{2}\right)=0
$$

where in the last equality, we exploited that the two tachyon vertex operators are on-shell and have equal mass. The amplitude, which at first sight looked like a gauge dependent off-shell quantity (because of the factor with explicit dependence on the insertion points $z_{i}$ ), upon closer inspection is equal to zero. Both in the contour argument and in the explicit calculation, there is no constraint on the momentum $k_{1}$ of the pure gauge vertex operator. The ghost structure of the vertex operator is of primordial importance in both arguments. Our calculation is an elementary illustration of the fact that BRST closedness (or in this case, exactness) is sufficient for a vertex operator to be on-shell. This fact is important in the bulk of the paper since we wish to work with arbitrary gauge parameters $\xi$. Our story here is but a close-up of a more systematic, general, and abstract treatment within for instance closed string field theory [2]. 


\section{B Factorization and doubling}

T-duality exchanges metric and anti-symmetric tensor components, and exchanges diffeomorphisms for anti-symmetric gauge transformations. In section 2 , the two types of transformations were on a different footing. T-duality (see e.g. [29]) feeds the desire to treat them democratically. In order to do so, it is convenient to double the number of space-time coordinates (see e.g. [10] for an introduction to the relevant concepts). We wish to introduce a left and a right space-time coordinate $X^{L}$ and $X^{R}$, as well as their shifts $\xi^{L}$ and $\xi^{R}$. There are now a number of different ways to proceed.

We wish to entertain the possibility to obtain an $O(d, d)$ invariant algebra as a result of our procedure. To do so, we can make the guess that the second seed on which our deformed BRST charge acts should be of the form:

$$
s_{2}=2 c \xi^{L 2} \cdot \partial X-2 \tilde{c} \xi^{R 2} \cdot \bar{\partial} X
$$

Note how this is in contrast to what we did in section 2 . We have flipped the left and right component of the second seed (only). This puts a particular twist on our gauge parameters. This idea leads to the following proposal for the BRST exact shift of the background after performing transformations 1 and 2 consecutively:

$$
\begin{aligned}
C_{12}=2 c \tilde{c}\left(\bar{\partial} \xi^{2 L}\left(X^{L}+\xi^{1 L}, X^{R}+\xi^{1 R}\right)\right. & \cdot \partial\left(X+\xi^{1 L}\right) \\
& \left.+\partial \xi^{2 R}\left(X^{L}+\xi^{1 L}, X^{R}+\xi^{1 R}\right) \cdot \bar{\partial}\left(X+\xi^{1 R}\right)\right) .
\end{aligned}
$$

Given this starting point, it is straightforward to calculate the commutator. We expand to second order in $\xi$, using amongst others the rule:

$$
\xi^{L 2}\left(X^{L}+\xi^{L 1}, X^{R}+\xi^{R 1}\right) \approx \xi^{L 2}+\xi^{L 1 \rho} \partial_{\rho}^{L} \xi^{L 2}+\xi^{R 1 \rho} \partial_{\rho}^{R} \xi^{L 2}+\ldots
$$

The full commutator can be summarized as:

$$
\begin{aligned}
C=2 c \tilde{c}[- & \partial \xi^{L 2} \cdot \bar{\partial} \xi^{L 1}+\partial \xi^{L 1} \cdot \bar{\partial} \xi^{L 2}-\bar{\partial} \xi^{R 2} \cdot \partial \xi^{R 1}+\bar{\partial} \xi^{R 1} \cdot \partial \xi^{R 2} \\
& +\bar{\partial}\left(\xi^{L 1 \rho} \partial_{\rho}^{L} \xi^{L 2}-\xi^{L 2 \rho} \partial_{\rho}^{L} \xi^{L 1}+\xi^{R 1 \rho} \partial_{\rho}^{R} \xi^{L 2}-\xi^{R 2 \rho} \partial_{\rho}^{R} \xi^{L 1}\right) \cdot \partial X \\
& \left.+\partial\left(\xi^{L 1 \rho} \partial_{\rho}^{L} \xi^{R 2}-\xi^{L 2 \rho} \partial_{\rho}^{L} \xi^{R 1}+\xi^{R 1 \rho} \partial_{\rho}^{R} \xi^{R 2}-\xi^{R 2 \rho} \partial_{\rho}^{R} \xi^{R 1}\right) \cdot \bar{\partial} X\right]
\end{aligned}
$$

We wish to express the commutator in terms of the gauge parameters. To that end, we need to decide how we will interpret the first line in (B.4). We rewrite the first two terms in the first line as:

$$
\begin{gathered}
C_{L 1}=-c \tilde{c}\left(\partial\left(\xi^{L 2} \cdot \bar{\partial} \xi^{L 1}\right)-\bar{\partial}\left(\xi^{L 2} \cdot \partial \xi^{L 1}\right)+\bar{\partial}\left(\partial \xi^{L 2} \cdot \xi^{L 1}\right)-\partial\left(\xi^{L 1} \cdot \bar{\partial} \xi^{L 2}\right)\right) \\
=-c \tilde{c}\left(\partial\left(\xi^{L 2} \cdot \partial_{\mu}^{R} \xi^{L 1} \bar{\partial} X^{\mu}\right)-\bar{\partial}\left(\xi^{L 2} \cdot \partial_{\mu}^{L} \xi^{L 1} \partial X^{\mu}\right)\right. \\
\left.+\bar{\partial}\left(\partial_{\mu}^{L} \xi^{L 2} \cdot \xi^{L 1} \partial X^{\mu}\right)-\partial\left(\xi^{L 1} \cdot \partial_{\mu}^{R} \xi^{L 2} \bar{\partial} X^{\mu}\right)\right) .
\end{gathered}
$$


Using this rewriting, we find the commutators of gauge parameters:

$$
\begin{gathered}
{\left[\left(\xi_{L 1}, 0\right),\left(\xi_{L 2}, 0\right)\right]=2\left(\xi^{L 1 \rho} \partial_{\rho}^{L} \xi^{L 2}-\xi^{L 2 \rho} \partial_{\rho}^{L} \xi^{L 1}, 0\right)} \\
\quad+\left(\xi^{L 2} \cdot \partial_{\mu}^{L} \xi^{L 1}-\xi^{L 1} \cdot \partial_{\mu}^{L} \xi^{L 2},-\xi^{L 2} \cdot \partial_{\mu}^{R} \xi^{L 1}+\xi^{L 1} \cdot \partial_{\mu}^{R} \xi^{L 2}\right) \\
{\left[\left(\xi_{L 1}, 0\right),\left(0, \xi_{R 2}\right)\right]=2\left(-\xi^{R 2 \rho} \partial_{\rho}^{R} \xi^{L 1}, \xi^{L 1 \rho} \partial_{\rho}^{L} \xi^{R 2}\right) .}
\end{gathered}
$$

On the right hand side, we read off the left parameter from the $\partial X$ terms, in accordance with the twist implemented in the second seed (B.1). We have succeeded in twisting our construction such that the resulting algebra gives rise to the $O(d, d)$ invariant Cbracket $[10,15,16]$. This bracket also has a world sheet vertex operator algebra counterpart which exibits $\alpha^{\prime}$ corrections found in [7].

We stress that we have improvised at the level of a twist on the space of gauge parameters, and the proposal for the resulting BRST exact state. It would be interesting to find a good rationale behind the construction of the C-bracket in this BRST covariant context.

Open Access. This article is distributed under the terms of the Creative Commons Attribution License (CC-BY 4.0), which permits any use, distribution and reproduction in any medium, provided the original author(s) and source are credited.

\section{References}

[1] R.P. Feynman, F.B. Morinigo, W.G. Wagner and B. Hatfield, Feynman lectures on gravitation, Addison-Wesley, Reading U.S.A. (1995).

[2] B. Zwiebach, Closed string field theory: quantum action and the B-V master equation, Nucl. Phys. B 390 (1993) 33 [hep-th/9206084] [INSPIRE].

[3] D. Ghoshal and A. Sen, Gauge and general coordinate invariance in nonpolynomial closed string theory, Nucl. Phys. B 380 (1992) 103 [hep-th/9110038] [INSPIRE].

[4] A. Giveon, D. Kutasov and N. Seiberg, Comments on string theory on $A d S_{3}$, Adv. Theor. Math. Phys. 2 (1998) 733 [hep-th/9806194] [INSPIRE].

[5] J. Troost, The AdS 3 central charge in string theory, Phys. Lett. B 705 (2011) 260 [arXiv: 1109.1923] [INSPIRE].

[6] G. Barnich and C. Troessaert, Aspects of the BMS/CFT correspondence, JHEP 05 (2010) 062 [arXiv: 1001.1541] [INSPIRE].

[7] W. Schulgin and J. Troost, Asymptotic symmetry groups and operator algebras, JHEP 09 (2013) 135 [arXiv:1307.3423] [INSPIRE].

[8] A. Strominger, On BMS invariance of gravitational scattering, JHEP 07 (2014) 152 [arXiv: 1312.2229] [INSPIRE].

[9] Y. Geyer, A.E. Lipstein and L. Mason, Ambitwistor strings at null infinity and subleading soft limits, arXiv:1406.1462 [INSPIRE].

[10] B. Zwiebach, Double field theory, T-duality and Courant brackets, Lect. Notes Phys. 851 (2012) 265 [arXiv:1109.1782] [INSPIRE].

[11] O. Hohm, D. Lüst and B. Zwiebach, The spacetime of double field theory: review, remarks and outlook, Fortsch. Phys. 61 (2013) 926 [arXiv:1309.2977] [INSPIRE]. 
[12] A.S. Losev, A. Marshakov and A.M. Zeitlin, On first order formalism in string theory, Phys. Lett. B 633 (2006) 375 [hep-th/0510065] [INSPIRE].

[13] O. Hohm, W. Siegel and B. Zwiebach, Doubled $\alpha^{\prime}$-geometry, JHEP 02 (2014) 065 [arXiv: 1306.2970] [INSPIRE].

[14] M. Evans and B.A. Ovrut, Deformations of conformal field theories and symmetries of the string, Phys. Rev. D 41 (1990) 3149 [InSPIRE].

[15] W. Siegel, Superspace duality in low-energy superstrings, Phys. Rev. D 48 (1993) 2826 [hep-th/9305073] [INSPIRE].

[16] C. Hull and B. Zwiebach, The gauge algebra of double field theory and Courant brackets, JHEP 09 (2009) 090 [arXiv:0908.1792] [INSPIRE].

[17] B.H. Lian and G.J. Zuckerman, New perspectives on the BRST algebraic structure of string theory, Commun. Math. Phys. 154 (1993) 613 [hep-th/9211072] [INSPIRE].

[18] V. Kac, Vertex algebras for beginners, American Mathematical Society, Providence, U.S.A. (1996).

[19] A.M. Zeitlin, Beta-gamma systems and the deformations of the BRST operator, J. Phys. A 42 (2009) 355401 [arXiv:0904.2234] [INSPIRE].

[20] A.M. Zeitlin, Beltrami-courant differentials and $G_{\infty}$-algebras, arXiv:1404.3069 [INSPIRE].

[21] A. Sen, On the background independence of string field theory, Nucl. Phys. B 345 (1990) 551 [INSPIRE].

[22] M. Campbell, P.C. Nelson and E. Wong, Stress tensor perturbations in conformal field theory, Int. J. Mod. Phys. A 6 (1991) 4909 [inSPIRE].

[23] B.A. Ovrut and S. Kalyana Rama, Deformations of superconformal field theories and target space symmetries, Phys. Rev. D 45 (1992) 550 [INSPIRE].

[24] K. Ranganathan, Nearby CFTs in the operator formalism: the role of a connection, Nucl. Phys. B 408 (1993) 180 [hep-th/9210090] [INSPIRE].

[25] K. Ranganathan, H. Sonoda and B. Zwiebach, Connections on the state space over conformal field theories, Nucl. Phys. B 414 (1994) 405 [hep-th/9304053] [INSPIRE].

[26] M. Cederwall, A. von Gussich and P. Sundell, Deformations in closed string theory: canonical formulation and regularization, Nucl. Phys. B 464 (1996) 117 [hep-th/9504112] [INSPIRE].

[27] O. Hohm and B. Zwiebach, Green-Schwarz mechanism and $\alpha^{\prime}$-deformed Courant brackets, arXiv: 1407.0708 [INSPIRE].

[28] J. Polchinski, String theory. Volume 1: an introduction to the bosonic string, Cambridge University Press, Cambridge U.K. (1998).

[29] A. Giveon, M. Porrati and E. Rabinovici, Target space duality in string theory, Phys. Rept. 244 (1994) 77 [hep-th/9401139] [INSPIRE]. 